\title{
Vibration exposure and work-musculoskeletal disorders among traffic police riders in Malaysia: a review
}

\begin{abstract}
Background: The traffic police force is one of the occupations that utilize motorcycles as the main mode of transport. The main ergonomic hazard with the constant use of motorcycles is exposure of riders to vibration while riding their motorcycles, which can potentially lead to work.related musculoskeletal disorders (WMSDs).

Objective: This review is meant to provide an overview of the available vibration exposure with WMSDs for traffic police riders and to review the related guidelines.

Method: This study involved comprehensive search of database from 1945 to 2015 . Published research paper that reported on the vibration exposure, prevalence, and/or risk factors of WMSDs and occupational riders were assessed and reviewed.

Result: The review suggests that the main factors involving vibration in motorcycles come from the motorcycles itself, the posture of the hands and arms during the gripping of the handlebars, the surrounding environment, and the period of riding motorcycles. Majority of the studies agreed that police riders had higher percentage of WMSDs especially in lower back, neck, and shoulder.

Conclusion: Overall, police riders have a high incidence of WMSDs, which in combination with exposure to vibrations with prolonged sitting and static posture may increase their susceptibility to WMSDs. Further research is required to explore the level of exposure to vibrations and WMSDs among traffic police riders, its potentially consequences, and ways to reduce exposure and risk associated with vibrations and WMSDs.
\end{abstract}

Keyword: Riders; Traffic policemen; Vibration; Work.related musculoskeletal disorders 